\title{
Phase field simulation of dendrite growth in Al-Cu alloy during solidification process
}

Feng $\mathrm{Li}^{1,2, a}$, Zhong Junhe ${ }^{1, b}$, Lu Nini ${ }^{1, c}$, Gao Yalong ${ }^{1, d}$, An Guosheng ${ }^{1,2, e}$

${ }^{1}$ College of Materials and Engineering, Lanzhou University of Technology, Lanzhou 730050, China

${ }^{2}$ State Key Laboratory of Advanced Processing and Recycling of Non-ferrous Metals, Lanzhou

University of Technology, Lanzhou 730050, China

a14314854@qq.com,b1781692974@qq.com, ${ }^{c} 1062078380 @ q q . c o m,{ }^{d} 1806450028 @ q q . c o m,{ }^{e} 2581$

50823@qq.com

Key words: phase field method; directional solidification; anisotropy; supercooling degree; competitive growth

Abstract: Based on the three-dimensional phase field model, the competitive growth of columnar crystals under the conditions of different anisotropies and different subcooling degrees in the directional solidification process was simulated with the Al-2\%mole-Cu two element alloy as an example. The influence of anisotropy and supercooling on the columnar crystal competition growth was studied. The simulation results showed that with the increase of anisotropy, the growth degree of non preferred dendrites increased first and then decreased; with the increase of supercooling, the morphology of preferred dendrites increased, and two dendrites appeared on the primary dendrite, but the change of non preferred dendrite morphology was not obvious.

\section{Introduction}

Directional solidification is a coercive method in the process of solidification. A certain temperature gradient is set up in the solidified metal and the non solidified liquid metal, so that the liquid metal solidified in the opposite direction of the heat flow, and a columnar crystal [1]with a specific orientation can be obtained. The directional solidification was first established and perfected in the development of superalloy. Directional solidification can be used to eliminate the transverse grain boundary in the crystallization process and improve the unidirectional mechanical properties of the materials. At present, directional solidification technology has been widely used in aerospace, shipbuilding, national defense and other important areas.

The methods for numerical simulation of solidification microstructure include deterministic method, stochastic method and phase field method [2]. The phase field method is a common computer numerical simulation technique. It can combine the micro and macro scale in the solidification process to avoid the difficulty of tracking the solid / liquid interface, thus directly simulating the dendrite growth morphology and growth process under the effect of concentration and other external fields, and can also quantitatively study the flow field, supercooling, disturbance, and anisotropy. The influence of anisotropy and orientation field on dendrite growth and appearance and morphology has become a hot topic in the research field of solidification structure [3-5].In recent years, relative scholars at home and abroad have used the phase field method to study the dendrites in the directional solidification process. Wang et al. [6] simulated the dendrite morphology, liquid solute concentration and solid solution in the single directional and multi directional columnar dendrites in the $\mathrm{Al}-4 \% \mathrm{Cu}$ two element alloy supercooled melt. Kumar et al. [7] uses thin interface phase field simulation to study the influence of interface anisotropy on the orientation selection of dendritic growth during directional solidification of the alloy. Zhang et al. [8], based on the three-dimensional phase field model, studied the dendrites with the parameters of the supercooling and temperature gradient during the directional solidification of DD6 superalloy. The 
influence of three-dimensional growth morphology; Feng et al. [9] uses the three-dimensional phase field model to simulate the directional solidification process of $\mathrm{Al}-\mathrm{Cu}$ two element alloy. The change law of the solid liquid interface and the coarsening mechanism of the solid liquid interface in the directional solidification process are studied, and the influence of different supercooling degrees on the interface shape is analyzed.In the process of dendrite growth, under the influence of the solidification conditions, it is also restricted by the anisotropy and supercooling degree, in which the supercooling is the main factor, because the growth of the dendrite is the process of the continuous stacking of the atoms in the liquid phase to the solid liquid interface, and the diffusion rate of the atoms will be reduced and then the dendrite will be reduced at a low temperature. Growth rate decreases, anisotropy influences the growth behavior of dendrite to a great extent, [10-12]. At present, there are few reports on three-dimensional phase field simulation of dendritic growth under different undercooling and anisotropy at home and abroad.

Based on the three-dimensional phase field model, this paper takes the Al-2\%mole-Cu two element alloy as an example to simulate the competitive growth of columnar crystals under the different anisotropy and different supercooling conditions during directional solidification, and studies the influence of anisotropy and supercooling on the competitive growth of columnar crystals.

\section{The phase-field model of competitive growth of dendrites}

\section{The phase-field}

Here, the normal vector in the solid-liquid interface of dendrites in migration direction is defined as follow:

$$
\vec{n}=\nabla \phi \text {. }
$$

The phase-field governing equation described in the literature [13] is employed:

$$
\frac{\partial \varphi}{\partial t}=M\left(\varepsilon(\theta)^{2} \nabla^{2} \phi+\left(\varepsilon(\theta) \cdot \varepsilon^{\prime \prime}(\theta)+\varepsilon^{\prime}(\theta)^{2}\right) \cdot(|\nabla \phi| \nabla \vec{n})-f \phi\right) .
$$

where, $\varnothing, M$, and $t$ represent the order parameter of phase field, the migration rate of solid-phase interface, and the time variable, respectively. Moreover, $\varepsilon(\theta)$ and $f_{\varnothing}$ refer to the parameter related to the surface energy and the first-order derivative of free energy density with respect to the order parameter of the phase field, respectively. The latter can be expressed as follows:

$$
f_{\phi}=\frac{R T}{V m} h^{\prime}(\phi) \ln \frac{\left(1-C_{S}^{\varepsilon}\right)\left(1-C_{L}\right)}{\left(1-C_{L}^{\varepsilon}\right)\left(1-C_{S}\right)}+W g^{\prime}(\phi) \text {. }
$$

where $R, T, V m, h(\varphi), W, g(\varphi)$, and $c$ represent the universal gas constant, temperature, molar volume, potential function, phase-field parameter, residual free energy function, and solute concentration of the alloy, respectively. Moreover, subscripts L and S as well as superscript $e$ refer to the liquid phase, solid phase, and an equilibrium state, respectively.

$$
\varepsilon(\theta)=1-3 \varepsilon_{4}+4 \varepsilon_{4}\left(\cos ^{4} \theta_{1} \cos ^{4} \theta_{2}+\cos ^{4} \theta_{3}\right) .
$$

In the formula: $\theta_{1}, \theta_{2}$, and $\theta_{3}$ represent the three angles of the normal direction of the interface 
migration of the dendritic growth and the coordinate system respectively.

In this model, $\theta_{1}, \theta_{2}$, and $\theta_{3}$ need to meet the following requirement:

$$
\cos ^{2} \theta_{1}+\cos ^{2} \theta_{2}+\cos ^{2} \theta_{3}=1 \text {. }
$$

\section{Solute-field governing equations}

The governing equation describing the diffusion and redistribution of solute can be written as follows:

$$
\frac{\partial c}{\partial t}=\nabla\left(\frac{D(\phi)}{f_{c c}} \nabla f_{c}\right)
$$

where $D(\varphi)$ is the solute diffusion rate while $f_{c}$ and $f_{c c}$ represent the first- and second-order derivatives of free energy with respect to concentration, respectively.

Physical parameters of materials

By taking $\mathrm{Al}-2 \%$ mole- $\mathrm{Cu}$ alloy as an example, the competitive growth of dendrites in directional solidification process was investigated. The physical parameters of the alloy are summarised in Table 1.

Table 1 Material physical parameters

\begin{tabular}{cc}
\hline Physical parameters & $\mathrm{Al}-2 \%-\mathrm{mole}-\mathrm{Cu}$ \\
\hline Interfacial energy $\sigma,\left(\mathrm{J} \cdot \mathrm{m}^{-1}\right)$ & 0.093 \\
Melting temperature $T_{\mathrm{m}},(\mathrm{K})$ & 933.3 \\
Equilibrium constant $k^{e}$ & 0.14 \\
Latent heat of the solvent $\mathrm{L} /\left(\mathrm{kJ} \cdot \mathrm{Kg}^{-1}\right)$ & 389.0 \\
Diffusion coefficient of liquid-phase solute $D_{\mathrm{L}}$, & $3.0 \times 10^{-9}$ \\
$\left(\mathrm{~m}^{2} \cdot \mathrm{s}^{-1}\right)$ & $3.0 \times 10^{-13}$ \\
Diffusion coefficient of solid-phase solute $D \mathrm{~S}$, & 620 \\
$\left(\mathrm{~m}^{2} \cdot \mathrm{s}^{-1}\right)$ & $1.0547 \times 10^{-5}$ \\
\hline Liquidus slope $m^{e},\left(\mathrm{~K}^{-5} \mathrm{~mol}^{-1}\right)$ & \\
Molar volume $V_{\mathrm{m}},\left(\mathrm{m}^{3} \cdot \mathrm{mol}^{-1}\right)$ &
\end{tabular}

\section{Initial conditions and boundary conditions}

Equations (2), (9) are calculated simultaneously by using an explicit finite difference method, both of which apply the same time step $\Delta t$. Where, $\Delta t$ and special step $\Delta x(\Delta x=\Delta y=\Delta z)$ satisfy the following stable conditions:

$$
\Delta t<\min \left\{\Delta x^{2} /\left(6 D_{L}\right), \Delta y^{2} /\left(6 D_{L}\right), \Delta z^{2} /\left(6 D_{L}\right)\right\} .
$$

Where, $D_{L}$ refers to the diffusion coefficient of the liquid-phase solute.

In the simulation of three-dimensional calculation, the direction of temperature gradient is parallel to the $\mathrm{Z}$ axis, and the calculation area is $300 \times 100 \times 500$. The initial nucleation is set to $\mathrm{r}=6 \times 10^{-8} \mathrm{~m}$ hemisphere. In this paper, the effects of anisotropy and supercooling on the competitive growth of columnar crystals are studied with 6 grains as the research object. In this paper, the dendrites that parallel the direction of growth and the direction of the temperature gradient are preferred dendrites, such as the grain 3 and 4 in Figure 1 (the growth direction is shown in the white 
arrow in the figure); the dendrites with a certain angle $\theta$ in the direction of growth and the temperature gradient are non preferred dendrites, such as the grain 1 and 2 in Figure 1.

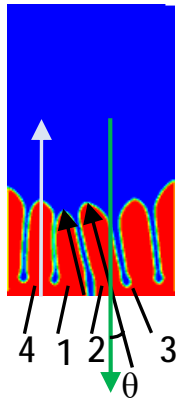

Figure 1 Picture of grain growth

\section{Results and analysis}

\section{The influence of anisotropy on the dendrite morphology}

Figure 2 shows the three-dimensional graph,slice of columnar crystal and the corresponding solute distribution diagram of columnar crystal under the different anisotropic conditions $(\mathrm{T}=25 \mathrm{k}, \theta$ $=15^{\circ}, \mathrm{t}=10000 \Delta \mathrm{t}$ ). Figure 2 (a-e) shows the growth morphology of the anisotropy of $0.055,0.060$, $0.065,0.070,0.075$, respectively; Figure 2 (a1-e1) shows a slice map parallel to the yoz plane, and the right color ruler is a scale for the phase order parameter value in Figure 2 (a1-e1); and Fig. 2 (a2-e2) is a solute distribution map of the corresponding figure 2 (a1-e1), right The edge color scale is a scale for the content of solute in Figure 2 (a2-e2).

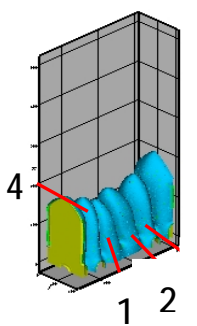

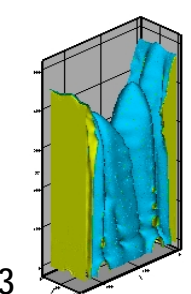

(b) $\delta=0.060$

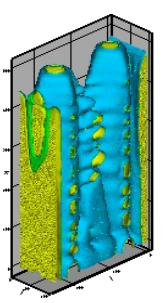

(c) $\delta=0.065$
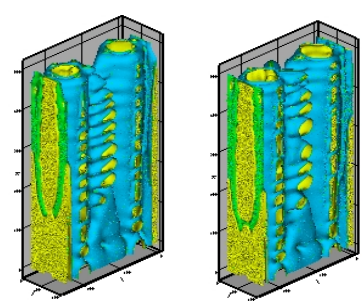

(d) $\delta=0.070--$ -

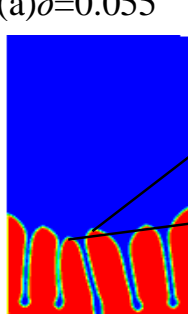

(a1)

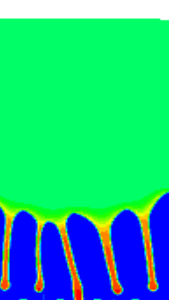

(a2)

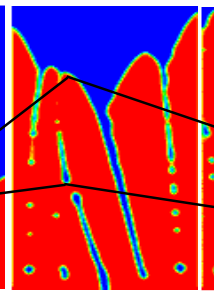

(b1)

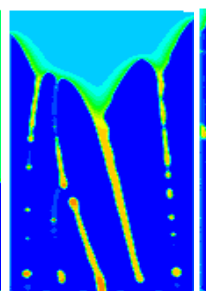

(b2)

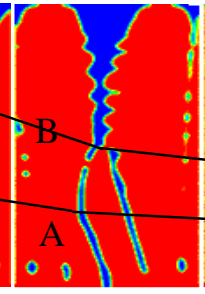

(c1)

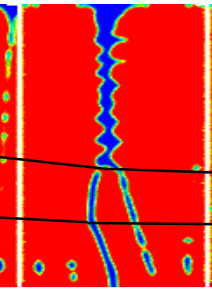

(d1)

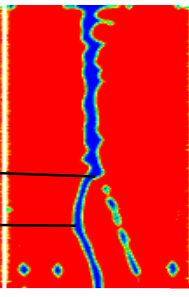

$(\mathrm{e} 1)$
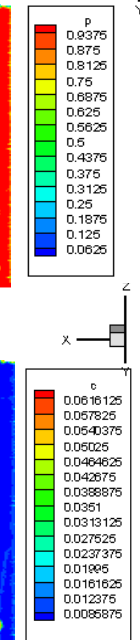

(e2)

Fig. 2 the morphology of dendritic growth under different anisotropic conditions.

When the anisotropy is 0.055 , the growth degree of the preferred dendrites 3,4 and the non preferred dendrites 1 and 2 is low, because the growth rate of the preferred dendrites and non 
preferred dendrites is very small, and the growth degree is not obvious, as shown in Figure 2 (a1). With the increase of anisotropy, the growth degree of non preferred dendrite 2 is larger than that of non preferred dendrite 1 , which is due to the greater inhibition of non preferred dendrite 1 and less growth rate, while the inhibition of non preferred dendrite 2 is smaller and the growth rate is larger, as shown in Figure 2 (b1). With the increasing anisotropy, the growth degree of non preferred dendrites 1 and 2 decreases with the increase of anisotropy, which is because when the anisotropy increases, the growth inhibition of the preferred dendrites on the growth of non preferred dendrites is increased, as shown in Figure 2 (c1-e1). At the same time, the enrichment of solute also hinders the growth of non preferred dendrites, and the concentration of solute is the highest in the liquid phase of the preferred dendrites, because the velocity of the precipitated solute diffusion in the liquid phase is less than the rate of dendrite growth, so that the precipitated solute can not diffuse into the liquid phase and form the solute enrichment. The growth rate of non preferred dendritic growth is decreasing, as shown in Figure 2 (b2-e2). It can be seen that in the process of dendrite growth, the preferred dendrites occupy the growth space of non preferred dendrites, and the non preferred dendrites are suppressed. With the increase of anisotropy, the growth degree of non preferred dendrites increases first and then decreases, as shown in the folding line A and B in Figure 2 .

\section{The influence of supercooling on the dendrite morphology}

Figure 3 shows the three-dimensional graph, slice map and the corresponding solute distribution diagram of columnar crystal growth under different supercooling conditions (anisotropic $\delta=0.070, \theta=15^{\circ}, \mathrm{t}=10000 \Delta \mathrm{t}$ ). Figure 3 (a-e) shows the growth morphology of $25 \mathrm{k}$, $27.5 \mathrm{k}, 30 \mathrm{k}, 32.5 \mathrm{k}, 35 \mathrm{k}$, respectively; Figure 3 (a1-e1) shows a slice map parallel to the yoz plane, and the right color ruler is the scale of the phase order parameter value in Figure 3 (a1-e1); Figure 3 (a2-e2) is a solute distribution map of the corresponding figure 3 (a1-e1), and the right color label the ruler is a scale equivalent to the content of solute in Figure 3 (a2-e2).

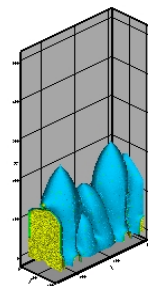

(a) $\Delta T=25 \mathrm{k}$

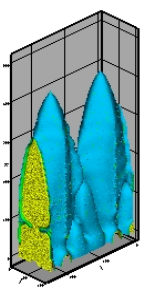

(b) $\Delta T=27.5 \mathrm{k}$

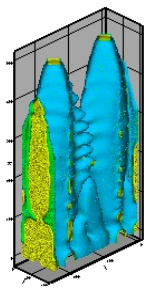

(c) $\Delta T=30 \mathrm{k}$

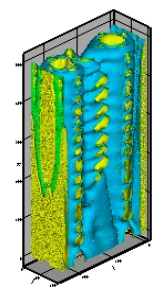

(d) $\Delta T=32.5 \mathrm{k}$

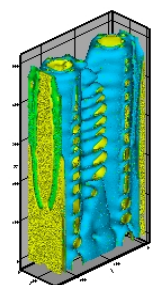

(c) $\triangle 130 \mathrm{k}$

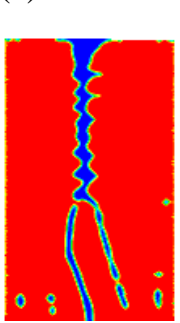

(c1)

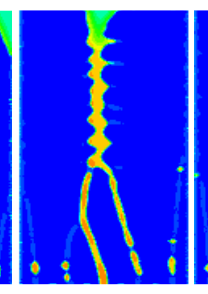

(c2)

(a2)
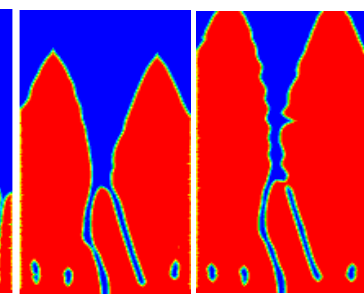

(b1)
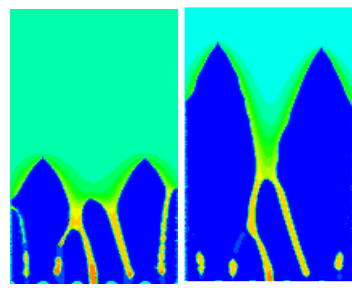

(b2)

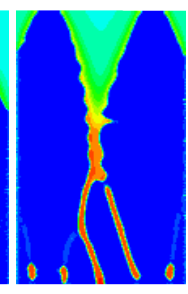

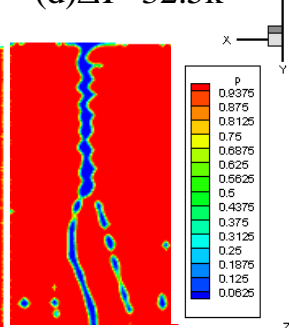

(d1)

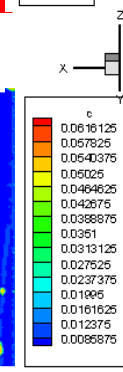

$(\mathrm{d} 2)$

Fig. 3 the morphology of dendritic growth under different undercooling conditions. 
When the supercooling degree is $25 \mathrm{k}$, it is observed that the preferred dendrite collar is prior to the non preferred dendrite growth, which is because the preferred dendrites are parallel to the temperature gradient growth, as shown in Figure 3 (a1). With the increase of the supercooling, the growth degree of the non preferred dendrites 1 and 2 varies greatly, because the selective dendrite 4 inhibits the non preferred dendrite 1 . The growth rate is very small and the growth is almost stopped, but the inhibition of non preferred dendrite 2 is smaller and can continue to grow, as shown in Figure 3 (b1). With the further increase of supercooling, the growth morphology of preferred dendrites is obviously increased, but the growth morphology of non preferred dendrites remains unchanged, which is because the preferred dendrites are born with time. The growth of non preferred dendrites was inhibited, and the concentration of solute precipitated from the two sides of the preferred dendrites resulted in the reduction of the actual supercooling degree, thus inhibiting the growth of non preferred dendrites, as shown in Figure 3 (c1-e1). It can be seen that with the increase of the cooling degree, the growth rate of the preferred dendrite increases gradually, the stability of the interface decreases, and two dendrites appear on the primary dendrite, while the non preferred dendrite growth rate remains unchanged, and the change degree of the morphology is not obvious.

\section{Conclusions}

In this paper, a $\mathrm{Al}-2 \%$ mole-Cu two element alloy is taken as an example to simulate the competitive growth of columnar crystals under different anisotropy and supercooling conditions during directional solidification, and the influence of anisotropy and supercooling on the competitive growth of columnar crystals is studied. The main rules are as follows:

(1) with the increase of anisotropy, the growth degree of non preferred dendrite increased first and then decreased.

(2) with the increase of supercooling degree, the preferred dendrite growth morphology increased, and the two dendrites appeared on the primary dendrite backbone, but the degree of non preferred dendritic morphology did not change significantly.

\section{References}

[1] Y. Shibuta, M. Ohno, T. Takaki. Solidification in a supercomputer: From crystal nuclei to dendrite assemblages. JOM, 2015, 67(8): 1793-1804.

[2] Shi Hu , Wen Xi, Zheng Chen, Song Wang, Ting-Hui Zhang.Computational Materials Science . 132 (2017):125-131.

[3] Li Yong,Li Huan,Zhao Ya ru,Zhou Ya ting.Research Progress in Phase Field Simulation of Directional Solidification Microstructre.JiangXi University of Science and Technology. (2016) 95-104.

[4] Feng Li, Jia Beibei, Zhu Changsheng, et al. Phase field simulation of multiple phase transformation of Fe-C alloy during solidification process. Measurement and intelligent materials, 2016,43: 855-860.

[5] Seong Gyoon Kim, Won Tae Kim and Toshio Suzuki. Phase-field model for binary alloys. Physical Review E, 1999, 60 (6):7186-7197.

[6] J. Kundin, L. Mushongera, H. Emmerich. Phase-field modeling of microstructure formation during rapid solidification in Inconel 718 superalloy. Acta Materialia, 2015, 95: 343-356. 
[7] Y. Li, P, J, Zheng, J, B, Zhang, Y, Liu, X, T, Yang, Research progress and prospect of directional solidification technology.Materials Review A, 2014,28(12):108-112.

[8] Liu Lin, Huang Taiwen, Qu Min, et al. High thermal gradient directional solidification and its application in the processing of nickel based super alloys. J Mater Process Techn,2010,210(1):159.

[9] FENG Li, HU Hai-huang, ZHU Chang-sheng, et al. Three-dimensional phase-field simulation of directional solidification of binary alloy. Journal of Lanzhou University of Technology, 2016,42(1):11-16.

[10] M. Yang, S. M. Xiong, Z. Guo. Characterisation of the 3-D dendrite morphology of magnesium alloys using synchrotron X-ray tomography and 3-D phase-field modelling. Acta Materialia, 2015, 92: 8-17.

[11] P. Steinmetz, Y. C. Yabansu, J. Hoetzer. Analytics for microstructure datasets produced by phase-field simulations. Acta Materialia, 2016, 103: 192-203.

[12] W.Wang,P.D.Lee, M,Mclean,A model of solidification microstructures in nickel-basde superalloys:predicting primary dendirte spacing selection ,Acta Mater 51(2003)2971-2987. 